\title{
EL BAYESIANISMO: DE LO CASUÍSTICO A LO HISTÓRICO
}

\author{
Milton Enrique Quero-Virla ${ }^{1}$ \\ https://orcid.org/0000-0003-4398-4911
}

\begin{abstract}
RESUMEN
Este documento presenta fundamentalmente dos aspectos relacionados al asunto Bayesiano: i) dos casos de aplicación de la epistemología Bayesiana, y, ii) una exposición resumida e intuitiva de la formalidad de las técnicas Bayesianas y su núcleo, el teorema de Bayes, culminando con una breve reseña histórica y una concepción de la epistemología Bayesiana. Apoyándose en la metodología de revisión documental bibliográfica, una caracterización general de un contexto de análisis Bayesiano describe dos situaciones cognitivas comprensivas de ese contexto, la inferencia Bayesiana y la teoría de decisión Bayesiana; además, se comentan aplicaciones Bayesianas generales y luego se dan dos casos específicos de aplicación: en el ejercicio médico cotidiano, y en la "plani-evaluación” de proyectos sociales. Una breve sección expone de manera muy básica las formalidades metodológicas Bayesianas interpretando el teorema de Bayes y el concepto de verosimilitud. Finalmente, una sección de cierre incluye una reseña histórica y una definición integradora de los principales componentes de la epistemología Bayesiana. La consolidación de epistemología Bayesiana ha requerido el aporte de muchas autoridades en el asunto; dos de los más influyentes pioneros mencionados en este documento son Aykac y Brumat (1977) y Lindley (1977). Como conclusión, la epistemología Bayesiana se presenta como una teoría de aprendizaje en incertidumbre respecto a eventos o estados aleatorios e inciertos de las cosas, de la naturaleza o de la realidad, aprendizaje o nuevo conocimiento que es expresado en términos de probabilidades.
\end{abstract}

Palabras clave: epistemología Bayesiana, inferencia Bayesiana, teoría de decisión Bayesiana, teorema de Bayes, verosimilitud.

\section{O Bayesianismo: Do casuístico ao bistórico.}

\section{RESUMO}

Este documento apresenta fundamentalmente dois aspetos relacionados ao assunto Bayesiano: i) dois casos de aplicação da epistemologia Bayesiana, e, ii) uma exposição resumida e intuitiva da formalidade das técnicas Bayesianas e seu núcleo, o teorema de Bayes, terminando com uma breve resenha histórica e uma concepção da epistemologia Bayesiana. Se sustenta na metodologia de revisão documental bibliográfica, uma caracterização geral de um contexto de análises Bayesiano descreve dois situações cognitivas compreensivas de esse contexto, a inferência Bayesiana e a teoria da decisão Bayesiana; ademais, se comentam aplicações Bayesianas gerais e depois se dão dois casos específicos de aplicação: no exercício médico cotidiano, e na "plani-avaliação" de projetos sociais. Uma breve seção expõe de maneira muito básica as formalidades metodológicas Bayesianas interpretando o teorema de Bayes e o conceito de verosimilitude. Finalmente, uma seção de fecho inclui uma resenha histórica e uma definição integradora dos principais componentes da epistemologia Bayesiana. A consolidação da epistemologia Bayesiana tem requerido o aporte de muitas autoridades no assunto; dois dos mais importantes pioneiros mencionados neste documento são Aykac e Brumat (1977) e Lindley (1977). Como conclusão, a epistemologia Bayesiana se presenta como uma teoria de aprendizagem com incerteza respeito a eventos ou estados aleatórios e incertos das coisas, da natureza ou da realidade, aprendizagem ou novo conhecimento que é expressado em términos de probabilidades.

Palavras chave: epistemologia Bayesiana, inferência Bayesiana, teoría da decisão Bayesiana, teorema de Bayes, verosimilitude.

Bayesianism: From the casuistic to the historical.

\section{ABSTRACT}

This document fundamentally presents two aspects related to the Bayesian matter: i) two cases of application of Bayesian epistemology, and, ii) a summarized and intuitive exposition of the formality of Bayesian techniques and its core, Bayes' theorem, culminating with a brief historical review and a conception of Bayesian epistemology., Based on documentary analysis and bibliographic review method, a general characterization of a Bayesian analysis context describes two comprehensive cognitive situations of that context, the Bayesian inference and the Bayesian decision theory; In addition, general Bayesian applications are discussed and then two specific cases of application are given: in daily medical practice, and in the "plan-evaluation" of social projects. A short section in a very basic way exposes Bayesian methodological formalities by interpreting Bayes' theorem and the concept of likelihood. Finally, a closing section includes a historical overview and an integrative definition of the main components of Bayesian epistemology.

${ }^{1}$ Universidad del Zulia. Maracaibo, Venezuela. Correo electrónico: miltonqv@gmail.com 
The consolidation of Bayesian epistemology has required the contribution of many authorities in the matter; two of the most influential pioneers mentioned in this document are Aykac and Brumat (1977) and Lindley (1977). In conclusion, Bayesian epistemology is presented as a theory of learning in uncertainty regarding random and uncertain events or states of things, of nature or reality, learning or new knowledge that is expressed in terms of probabilities.

Keywords: Bayesian epistemology, Bayesian inference, Bayesian decision theory, Bayes' theorem, likelihood.

\section{El contexto Bayesiano.}

De manera general, dentro de un entorno Bayesiano se incluye el problema básico de determinar un "estado de la naturaleza" subyacente, que es inicialmente incierto de alguna manera, y sobre la base de evidencia y contra-evidencia muestral subjetiva y/u objetiva, cambia a un estado de nuevo conocimiento o aprendizaje, sea en forma de inferencias, o como decisiones sobre cursos de acción. Este aprendizaje puede revisarse o actualizarse permanentemente siempre a la luz de nueva evidencia (Quero y Romero, 2016).

Con mayor precisión, dos situaciones cognitivas comprenden el contexto propio de estudio y aplicación de los asuntos Bayesianos: una, las de inferencia estadística, la otra, aquellas enmarcadas dentro del campo de la teoría de decisión estadística (Morgan, 1971, p.11). Los primeros incluyen acciones de aceptación o rechazo de alguna hipótesis, mientras los segundos incluirán la ejecución de varias decisiones posibles cuyas consecuencias dependen de estados desconocidos de la naturaleza.

Estas dos categorías de la llamada estadística Bayesiana, estrechamente relacionadas, la inferencia Bayesiana y la teoría de decisión Bayesiana, aún más precisamente tratan, la primera, con la estimación y la comprobación estadística, mediante información anterior subjetiva o información subjetiva y empírica, sin considerar formalmente "funciones de pérdida" asociadas con decisiones erróneas; mientras que la segunda, abarca principios y procedimientos para escoger un acto óptimo en una situación dada, basándose en cualquiera y todas las informaciones con funciones de pérdida formalmente expresadas y explícitamente evaluadas (Chou, 1978, p.360).

Chou, además, enfatiza en el asunto esencial de que una inferencia Bayesiana puede hacerse sobre información anterior solamente o sobre información anterior y una muestra; anterior en el sentido de que es la información que tiene el estadístico o decisor sobre un problema inferencial antes del muestreo. Esta información se incorpora en el análisis en términos probabilistas. Los Bayesianos siguen una interpretación subjetiva de la probabilidad, que representa "grados de creencia" de un individuo en distintas proposiciones, es decir, que la información anterior consiste a menudo en juicios personales acerca de una situación. Sin embargo, la aceptación de la interpretación subjetiva de la probabilidad no impide a los Bayesianos formular sus grados de creencia sobre la base de datos anteriores o del supuesto clásico de resultados igualmente probables, o de ambas cosas a la vez (Chou, 1978, p.360).

La estadística Bayesiana es, si se quiere, la contraparte de la llamada estadística clásica o "frecuentista", un universo paralelo al de la estadística clásica, con técnicas homólogas a todas las de esta, y posiblemente con mayor número de aplicaciones (Quero y Romero, 2016); no obstante, el método Bayesiano puede considerarse como una extensión del método clásico (Chou, 1978, p.361). Quizás desde la década de los años 90, la estadística Bayesiana ha pasado a ocupar un lugar propio dentro de la literatura estadística, en cuanto a libros de textos completos se refiere, superando el modesto espacio de una sección dentro de un capítulo, o a lo sumo un capítulo, que generalmente ocupaban dentro de los libros populares de estadística disponibles en las librerías del mercado y bibliotecas.

Ese hecho ha sido quizás una consecuencia de las ya numerosas aplicaciones que de la estadística Bayesiana se han dado; aplicaciones que van desde investigaciones que incluyen tratamientos muy cuantitativos, hasta aquellas de procesamientos más cualitativos, y pueden abordarse desde un enfoque Bayesiano. Entre ambos tipos de aplicaciones encontramos, por ejemplo, desde las que se apoyan en los diagramas de influencias, "ayudas gráficas cuali-cuantitativas alternativas a los más populares árboles de decisión, para tratar situaciones de decisiones secuenciales" (Quero, 2003), (Jensen, 1998), con el apoyo de programas computacionales específicos y que han conseguido su asidero en distintas áreas científicas; hasta las que pudieran tener un uso menos complejo, aunque 
igualmente importantes, de las técnicas o análisis Bayesiano, como en la planificación y evaluación de proyectos sociales o comunitarios (Quero, 2013).

Muchas referencias pueden darse donde se citan áreas específicas de aplicación de las técnicas o análisis bayesiano, pero se resumirán acá las expuestas por Silva (2009): La cantidad de ejemplos de índole muy diversa donde se aplica el enfoque bayesiano es abrumadora (...): Epidemiología moderna, Ensayos clínicos (adaptive clinical trials), Detección de correos electrónicos espurios (spam), Desarrollo de sistemas expertos para diagnóstico y pronóstico de enfermedades (...) redes bayesianas, Suavizamiento de mapas de morbilidad y mortalidad (Silva, 2009, pp. 450-454). Adicionalmente, es oportuno comentar, que títulos amplios de libros como: análisis Bayesiano para las ciencias sociales, bioestadística Bayesiana, estadística Bayesiana y mercadeo, métodos Bayesianos para ecología, métodos Bayesianos para dinámicas estructurales e ingeniería civil, econometría Bayesiana, métodos Bayesianos en finanzas, análisis de datos no paramétrico bayesiano, estadística Bayesiana no paramétrica y semi-paramétrica práctica, métodos Bayesianos en cosmología, y métodos Bayesianos para hackers, la mayoría escritos en idioma inglés, son ya comunes en el mercado internacional. Mediante una simple búsqueda por internet el lector puede corroborar la existencia de catálogos de libros en tiendas virtuales.

Corriendo el riesgo de menoscabar el valor de tantas importantes obras, se destaca acá la relevante contribución que pueden dar las relativamente recientes e interesantes obras: "Proving History: Baye's theorem and the quest for historical Jesus" por Richard Carrier (2012), en la cual se aplica la técnica y metodología Bayesiana para "elicitar" la probabilidad de ocurrencia de hechos históricos (Carrier, 2012), y, "The predictive mind", por Hohwy (2013), donde se relacionan conceptos de inferencia perceptual, estados de cosas, fenomenología, inferencia causal y métodos Bayesianos (Hohwy, 2013).

Para finalizar esta sección, si integramos las dos categorías de la estadística Bayesiana mencionadas al inicio, la inferencia y la teoría de decisión, y las catalogamos como técnicas Bayesianas, otra importante rama paralela a esas técnicas puede considerarse: el punto de vista Bayesiano, en cuyo estudio se muestran profundizaciones no solo en el nivel de las mencionadas técnicas Bayesianas, sino en los asuntos epistemológicos y esferas superiores de la filosofía de la ciencia. Algunos comentarios pueden revisarse en el Volumen 119 de Contribution to economic analysis (1977): "New developments in the applications of Bayesian methods", por Aykac y Brumat (Aykac y Brumat, 1977), (Gelman y Shalizi, 2013).

\section{Los médicos Bayesianos: Uno de los casos.}

Generalmente, de manera intuitiva, los "buenos" médicos en sus consultas asumen un razonamiento Bayesiano, aún sin tener una formación explícita en esta área, ni siquiera conocimiento del asunto Bayesiano. En su obra "Principios de la Teoría de la Decisión", Lindley (Lindley, 1977), traducida del original "Making decisions", 1971, por el Dr. J.M. Bernardo), ya describía con algunos detalles, cómo se da este ejercicio profesional, y se comenta a continuación. Cuando un paciente acude a consulta por primera vez, incluso antes de que entre al consultorio, el médico tendrá cierta idea de lo que puede pasarle. En efecto muchos pacientes tienen pequeños problemas como un resfriado, y unos pocos tendrán enfermedades "raras". Estás ideas pueden ser expresadas como probabilidades iniciales o a priori de que el paciente tenga una de esas enfermedades, las cuales son los sucesos o estados inciertos de la situación.

Una vez que el paciente entra a la consulta, describe sus síntomas, el médico hace preguntas, realiza pruebas y, en general, adquiere información, X. El médico debe procesar sus opiniones iniciales y $\mathrm{X}$ para producir sus opiniones a posteriori sobre lo que le pasa al paciente. Por otra parte, existe también una verosimilitud de ese conjunto de síntomas $\mathrm{X}$ en el paciente dado que realmente padece una determinada enfermedad de las consideradas; esta verosimilitud, sin mayor formalismo, podemos considerarla como una probabilidad condicional de que el conjunto de síntomas $\mathrm{X}$ esté asociado a una enfermedad específica. De esta manera, si una enfermedad específica $E$ es tal que ese conjunto de síntomas X está raramente asociado con ella, su verosimilitud será pequeña y, en 
consecuencia, su producto por la probabilidad inicial de la enfermedad será pequeño de forma que el médico concluirá que es poco probable que el paciente tenga esa enfermedad E. Además, si la probabilidad inicial de esa enfermedad específica $E$ es pequeña (como es el caso de una enfermedad rara o poco frecuente), incluso si el conjunto de síntomas $\mathrm{X}$ está a menudo asociado con esa enfermedad E, el producto será pequeño y el médico no asociará esa enfermedad E con el paciente, hasta que los síntomas sean muy claros o evidentes.

Lindley afirmó (Lindley, 1977), que lo que podía ocurrir, en resumen, es que en un futuro no muy lejano el médico dispondría en su consultorio de una computadora, introduciría la información X que obtiene, y el computador estaría programado para indicarle al médico, lo que probablemente esté padeciendo el paciente, es decir, las probabilidades a posteriori (Lindley, 1977). En la práctica, por lo menos desde los años 90, ya hay muestras concretas de estas aplicaciones en lo que se ha denominado como sistemas expertos Bayesianos en el área de la medicina (Díez, 1994).

\section{Otro caso: Los planificadores y gerentes Bayesianos.}

Consideremos como segundo caso la situación en la que unos "plani-evaluadores" socioeducativos con formación Bayesiana, desarrollan el Proyecto: Programa de Capacitación para una Comunidad, cuyos Objetivos son: 1.- Adquisición (Desarrollo) de Competencias; 2.Aprovechamiento Eficiente del Tiempo; los cuales pretenden alcanzar siguiendo las Estrategias de Capacitación: 1.- Estándar, con Consultas y Asesorías Regulares; y 2.- Reforzada, con Consultas y Asesorías Extras (Quero, 2014).

Supóngase además que los estados inciertos derivados de la ejecución del programa de capacitación son los indicados en la primera columna de la Figura 1: "Competencia Alta/Eficiencia Regular" (CA/ER), "Competencia Alta/Eficiencia Baja" (CA/EB), "Competencia Mediana/Eficiencia Regular" (CM/ER), y, "Competencia Mediana/Eficiencia Baja” (CA/EB). Al igual que en la sección anterior, del médico Bayesiano, el procedimiento de estimar las probabilidades posteriores de esos estados, requiere primeramente la estimación de las probabilidades anteriores (por ejemplo, según información histórica de programas aplicados en el pasado en condiciones similares); luego, la "elicitación" de las verosimilitudes de la información que los plani-evaluadores Bayesianos obtengan durante la ejecución del programa (v.g., la aplicación de pruebas de rendimiento y observaciones directas de ejecución, de los participantes que reciben la capacitación, lo cual constituye evidencia muestral).

Figura 1. Valoraciones Bayesianas de un Programa de Capacitación

\begin{tabular}{|c|c|c|c|c|}
\hline \multirow[b]{2}{*}{$\begin{array}{l}\text { ACCIÓN-EVENTO (Et) } \\
\text { CONSECUENCIAS }\end{array}$} & \multicolumn{4}{|c|}{ VALORACIONES BAYESIANAS (PROBABILIDADES) } \\
\hline & $\begin{array}{l}\text { PREVIAS } \\
\text { P(Et })\end{array}$ & $\begin{array}{c}\text { VEROSIIIILTUD } \\
\text { Evidancia (Ed) } \\
\text { P(Ed IEt) }\end{array}$ & $\begin{array}{c}\text { CONJUNTAS } \\
P(E t) \times P(E d \mid E t)\end{array}$ & $\begin{array}{c}\text { POSTERIORES } \\
\text { P (Et I Ed): CONJUNTA / } \\
\text { SJMA DE CONJUNTAS }\end{array}$ \\
\hline $\begin{array}{l}\text { Competencig Altg/ } \\
\text { Eficiencig Regulgr }\end{array}$ & $35 \%=0.35$ & $33 \%=0.33(1)$ & $0.35 \times 0.33=0.1155$ & $>0.1155 / 0.50=0,231=23.1 \%$ \\
\hline $\begin{array}{l}\text { Competencig Altg/ } \\
\text { Eficiencis Bsjg }\end{array}$ & $35 \%=0.35$ & $67 \%=0.67$ & $0.35 \times 0.67=0.2345$ & $0.2345 / 0.50=0,469=46.9 \%$ \\
\hline $\begin{array}{l}\text { Competencig Medisng/ } \\
\text { Eficiencig Regulgr }\end{array}$ & $15 \%=0.15$ & $11 \%=0.11$ & $0.15 \times 0.11=0.0165$ & $0.0165 / 0,50=0.033=3.3 \%$ \\
\hline \multirow[t]{2}{*}{$\begin{array}{c}\text { Competencis Medisng/ } \\
\text { Eficiencis Bsjg }\end{array}$} & $15 \%=0.15$ & $89 \%=0.89(2)$ & $0.15 \times 0.89=0.1335$ & $0.1335 / 0.50=0,267=26.7 \%$ \\
\hline & $\Sigma=100 \%=1$ & & $\Sigma=\%=50 \%=0.50$ & $\Sigma=100 \%=1$ \\
\hline
\end{tabular}

(1) De 30 Personas con CA, ER, en 10 ha predominado la Estrategia Estándar.

(2) De 45 Personas con CM, EB, en 40 ha predominado la Estrategia Reforzada.

Fuente: Quero (2014).

Asumiendo datos hipotéticos de la situación descrita, las probabilidades iniciales, las verosimilitudes, y los cálculos básicos para estimar las probabilidades posteriores de los estados 
inciertos y cursos de acción considerados, se muestran de forma tabular, en la ya referida Figura 1. Los símbolos usados en los encabezados de las columnas, permiten, intuitivamente, justificar los cálculos simples realizados. El análisis de las probabilidades posteriores a luz de las evidencias muestrales obtenidas, permite a los plani-evaluadores Bayesianos incorporar ajustes sucesivos en las estrategias de capacitación. Sin mayor profundidad, las probabilidades previas asociadas a los eventos de baja eficiencia tanto para competencia alta (CA) como para competencia mediana (CM), se han incrementado de $35 \%$ a $46.9 \%$, y de 15 a $26.7 \%$, respectivamente. Una de las interpretaciones-aprendizajes que pueden darse a estas actualizaciones de las probabilidades puede ser el que la implementación del Programa de Capacitación está requiriendo el empleo de una Estrategia Reforzada, con Consultas y Asesorías Extras.

\section{El formalismo Bayesiano.}

Lo que intuitivamente se ha inducido en los dos casos de aplicación del Bayesianismo en las dos secciones anteriores, no es más que el uso de un teorema de probabilidades condicionales conocido como el Teorema de Bayes.

Sin mucho rigor matemático, entre un conjunto estados inciertos o cursos de acción alternativos (o simplemente, alternativas) $\mathrm{E}_{\mathrm{i}}(\mathrm{i}=1, \ldots, \mathrm{n})$, definidos como eventos disjuntos o mutuamente excluyentes; y un evento A asociado a cierta información muestral relevante y pertinente que pudiera obtenerse, la probabilidad posterior de una alternativa $E_{j}$ cualquiera dado que se obtuvo información muestral A: $\mathrm{P}(\mathrm{Ej} \mid \mathrm{A})$ se obtiene mediante la expresión:

$$
P(E j \mid \mathrm{A})=\frac{\mathrm{P}(\mathrm{Ej}) \times \mathrm{P}(\mathrm{A} \mid \mathrm{Ej})}{\sum \mathrm{P}(\mathrm{Ei}) \times \mathrm{P}(\mathrm{A} \mid \mathrm{Ei})}
$$

Siendo $\mathrm{P}(\mathrm{Ei})$ la probabilidad previa de una alternativa $\mathrm{Ei}$, y $\mathrm{P}(\mathrm{A} \mid \mathrm{Ei})$, la verosimilitud del evento A dado que se siguió la alternativa Ei.

Además de la forma tabular que permite "operar" con datos usando el mencionado teorema, dos formas adicionales permiten graficar el uso del mismo: mediante un diagrama de Venn, y empleando un diagrama de árbol. Las tres formas se muestran en la Figura 2, y se han incluido unos datos ficticios, con el único propósito de ejemplificar los cálculos.

Figura 2. Formas gráficas y tabulares de interpretación y cálculos del Teorema de Bayes.

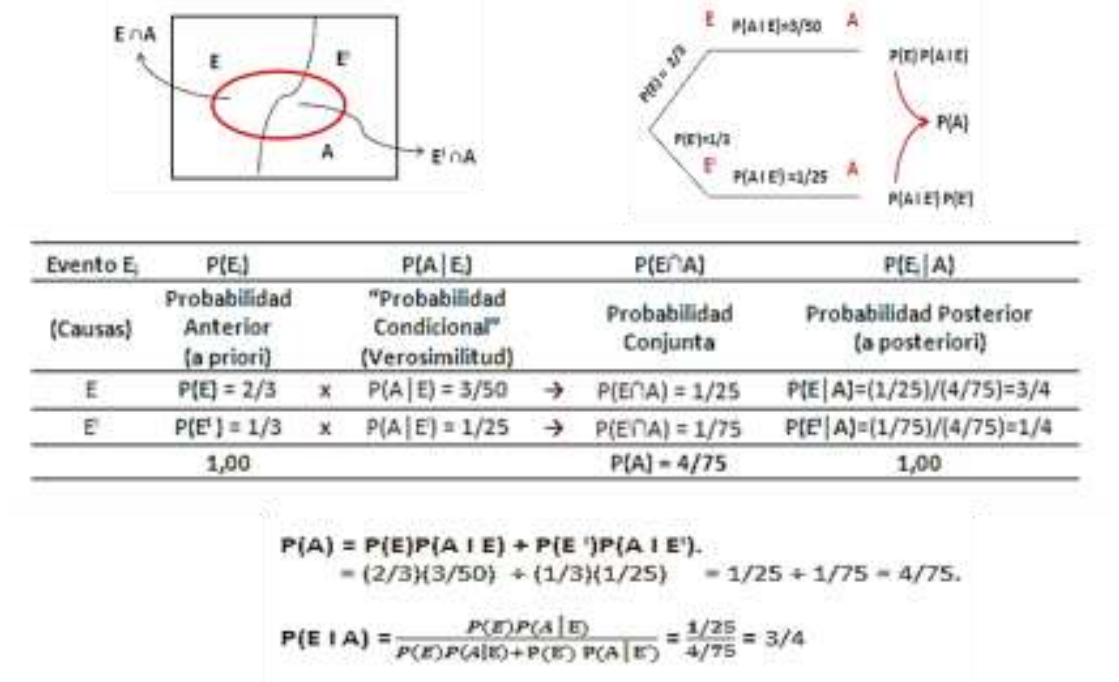

Específicamente, como en cualquier aplicación regular del Teorema de Bayes, las probabilidades subjetivas estimadas para los cursos de acción alternativos o eventos, es decir, las probabilidades 
previas, son aritméticamente multiplicadas por sus respectivas "verosimilitudes". Estas verosimilitudes son probabilidades condicionales, estimadas generalmente a partir de datos muestrales, probabilidades que con base en datos conocidos representan la credibilidad o "grados de creencia" de obtener un resultado o evidencia favorable a un "estado", "condición específica" o consecuencia, dado que se siguió un curso de acción alternativo determinado.

Los productos resultantes de las multiplicaciones anteriormente mencionadas, y denominados "probabilidades conjuntas", permiten obtener las "probabilidades posteriores" de cada curso de acción mediante el cociente de su probabilidad conjunta respectiva entre la suma de todas las probabilidades conjuntas correspondientes a todos los cursos de acción alternativos posibles, suma conocida como probabilidad total. Si se obtuviese información completa, una de las probabilidades previas se transformaría en una probabilidad posterior igual a uno (1); pero en la realidad lo que se obtiene es información parcial obteniendo así cambios menos acentuados en las probabilidades. A todas estas estimaciones de probabilidad o valoraciones probabilísticas, las hemos denominado "valoraciones bayesianas" (Quero, 2014).

\section{Reseña histórica y comentarios finales.}

Mayoritariamente se ha asumido que el Teorema de Bayes (también conocido como Regla de Bayes), es atribuible al Reverendo inglés Thomas Bayes (1702-1761), y fue publicado de manera póstuma en 1763 en el trabajo titulado "An essay toward solving a problema in the doctrine of chance" (Price, 1763).

En la actualidad, no obstante las posiciones detractoras, se considera que el Teorema de Bayes tiene una notable connotación práctica y epistemológica, y que su interés estriba en que por su conducto se estiman las probabilidades inversas de las hipótesis. En relación a esto se considera además, que la aproximación inferencial Bayesiana es, la más atractiva y promisoria, y que precisamente el enfoque Bayesiano se perfila como un nuevo paradigma inferencial para la estadística (Silva, 2009).

Las aplicaciones del Teorema de Bayes, y su estudio formal y sistemático, han derivado en un sólido cuerpo de principios y conocimientos, que bien pueden englobarse bajo el término Bayesianismo, o Epistemología Bayesiana, caracterizada como:

Una teoría de aprendizaje en incertidumbre con la cual personas, investigadores, científicos, individuales o colectivos, aprenden o conocen respecto a eventos o estados aleatorios $\mathrm{e}$ inciertos de las cosas, de la naturaleza, de la realidad, a partir de sus opiniones iniciales, creencias, juicios intuitivos, datos anteriores o históricos, o de considerar el supuesto clásico de resultados igualmente probables, expresados mediante probabilidades a priori subjetivas o grados de creencia -certeza o ignorancia relativa-, objetivas o de ambas, referidas a aquellos eventos o estados y que representan el conocimiento inicial o su parcial ignorancia; las que luego son ajustadas o revisadas, derivando en probabilidades a posteriori u opiniones finales, sobre la base de evidencias y contra-evidencias muestrales objetivas y subjetivas $-\mathrm{y}$ sus verosimilitudes asociadas- obtenidas mediante cualquier procedimiento fáctico de campo, intelectual o documental de recolección de información; probabilidades éstas que representan el nuevo conocimiento o aprendizaje acerca de aquel estado e evento incierto, sea en forma de inferencias, como de decisiones sobre cursos de acción. Este aprendizaje puede revisarse o actualizarse permanentemente siempre a la luz de nueva evidencia (Quero y Romero, 2016).

Menciones recientes, de esta misma década, muy importantes y que destacan la aceptación de los métodos Bayesianos en la comunidad científica son las publicadas por la Administración de Alimentos y Medicamentos (Food and Drug Administration) en el año 2010, y cuyos antecedentes se remontan a 1997 (Campbell, 2017), y por la Asociación Estadística Americana (American Statistical Association, ASA), en el año 2016 (Wasserstein \& Lazar, 2016).

Más allá de las connotaciones epistemológicas, algunas implicaciones filosóficas subyacen al Bayesianismo: 
1. Un resultado que deriva del Teorema de Bayes es que no es recomendable asignar probabilidades nulas a sucesos inciertos, puesto que si la probabilidad inicial es nula, también lo será la probabilidad final, cualesquiera que sean los datos (Lindley, 1977).

2. (...) Una probabilidad igual a uno es igualmente peligrosa, porque entonces la probabilidad complementaria sería cero. No crea usted en nada de forma absoluta (Lindley, 1977).

3. La posteriori de hoy pasa a ser la priori de mañana (Silva, 2009).

Referencias bibliográficas.

Aykac, Ahmet and Brumat, Carlo. (1977). New Developments in the Applications of Bayesian Methods: Proceedings of the First European Conference Sponsored by the Centre Européen D'éducation Permanente (CEDEP) and the Institut Européen D'administration Des Affaires (INSEAD), June 1976. Volumen 119 de Contributions to economic analysis. North-Holland Publishing Company. Amsterdam.

Campbell, Gregory. (2017). Bayesian methods in clinical trials with applications to medical devices. Communications for Statistical Applications and Methods. Vol. 24, No. 6, Korea. (Pp. 561-581). https://doi.org/10.29220/CSAM.2017.24.6.561

Carrier, Richard. (2012). Proving History: Baye's theorem and the quest for historical Jesus. Prometheus Books. USA.

Chou, Ya-Lun. (1978). Análisis estadístico. Interamericana. México.

Díez, Francisco. (1994). Sistema experto Bayesiano para ecocardiografía. Tesis doctoral. Doctorado en Ciencias Físicas. Universidad Nacional de Educación a Distancia. España.

Gelman, Andrew and Shalizi, Cosma. (2013). Philosophy and the practice of Bayesian statistics.

British Journal of Mathematical and Statistical Psychology. 66, England. (Pp. 8-38).

Hohwy, Jakob. (2013). The predictive mind. Oxford University Press. USA.

Jensen, Finn. (1998). An introduction to bayesian networks. Springer. Inglaterra.

Lindley, Dennis. (1977). Principios de la Teoría de la Decisión. Editorial Vicens-Vives. España. Morgan, Bruce. (1971). Introducción a los procesos bayesianos de decisión estadística. Paraninfo. España.

Price, Richard. (1763). An essay towards solving a problem in the doctrine of chances. By the Late Rev. Mr. Bayes, F. R. S. Communicated by Mr. Price, in a Letter to John Canton, A. M. F. R. S. Philosophical Transactions of the Royal Society, 53, England. (Pp. 370-418).

Quero, Milton y Romero, Nelson. (2016). Una aproximación Bayesiana entre el Objetivismo y el Subjetivismo. Revista Opción. Universidad del Zulia. Especial No. 3, Venezuela. (Pp. 301-326)

Quero, Milton. (2003). Toma de decisiones en dominios probabilísticos mediante diagramas de influencias. Trabajo de Grado. Magister Scientiae en Estadística Aplicada. Universidad de Los Andes, Venezuela.

Quero, Milton. (2013). Decisiones bayesianas integradas para la evaluación de proyectos comunitarios. Tesis Doctoral. Doctorado en Ciencias de la Educación. Universidad Rafael Belloso Chacín. Venezuela.

Quero, Milton. (2014). Decisiones Bayesianas integradas para la evaluación de proyectos comunitarios. Trabajo de Ascenso presentado para optar a la Categoría de Asociado. Facultad Experimental de Ciencias. Universidad del Zulia. Venezuela.

Silva, Luis. (2009). La investigación biomédica y sus laberintos. En defensa de la racionalidad para la ciencia del siglo XXI. Díaz de Santos. España.

Wasserstein, Ronald and Lazar, Nicole. (2016). The ASA's Statement on p-Values: Context, Process, and Purpose, The American Statistician,70:2, United States. (Pp. 129-133). DOI: https://dx.doi.org/10.1080/00031305.2016.1154108 